\title{
Prédispositions génétiques au sepsis sévère
}

\author{
S. Lavergne et J.-P. Mira
}

\section{Introduction}

Le sepsis est la première cause de décès en réanimation. Le pronostic de ce syndrome est lié à l'adéquation du traitement entrepris (antibiothérapie et mesures de réanimation rapides et adaptées), à l'agressivité du micro-organisme responsable de l'infection et au terrain de l'hôte (pathologies sous-jacentes : diabète, alcoolisme chronique, cirrhose...). En dehors de ces facteurs bien connus, il existe clairement une susceptibilité individuelle à l'infection qui pourrait expliquer pourquoi certains patients développent des tableaux cliniques dramatiques quand ils sont en contact avec un pathogène.

Les études animales et humaines ont apporté des preuves de l'existence de cette inégalité génétique face au risque infectieux. La résolution de la séquence génome humain, la compréhension des maladies génétiques mendéliennes et la connaissance des mécanismes de défense anti-infectieuse et de la physiopathologie du sepsis ont permis de cibler les gènes candidats pour l'étude des polymorphismes génétiques associés au sepsis.

\section{Preuves de l'existence d'une prédisposition génétique aux infections}

Les études expérimentales et les études épidémiologiques humaines de ces dernières décennies ont permis de démontrer le rôle des facteurs génétiques de l'hôte dans la survenue et/ou le pronostic des infections.

\section{Les études animales}

Les souris représentent un bon modèle d'étude sur l'infection car la plupart des gènes murins impliqués dans la réponse au sepsis ont des équivalents chez l'homme. Ainsi, si un déficit génétique est associé à une réponse anti-infec- 
tieuse anormale chez la souris, il est probable qu'un polymorphisme génétique humain qui modifierait la fonction de la protéine puisse être associé à une susceptibilité au même type d'infection.

Poltorak et al. ont ainsi étudié deux lignées de souris $(\mathrm{C} 3 \mathrm{H} / \mathrm{HeJ}$ issues de la lignée $\mathrm{C} 3 \mathrm{H} / \mathrm{HeN}$ et $\mathrm{C} 57 \mathrm{BL} / 10 \mathrm{ScCr}$ ) résistantes à l'injection d'une dose létale de lipopolysaccharide (LPS), qui est un des constituants principaux de la paroi de bactéries à Gram négatif. De façon intéressante, ces souris sont par ailleurs très sensibles aux infections à ces mêmes pathogènes. Ils ont mis en évidence des mutations ponctuelles du gène du récepteur Toll4, démontrant ainsi le rôle de ce récepteur dans la détection du LPS et dans la physiopathologie des infections à Gram négatif (1). Cependant, il est difficile d'affirmer à $100 \%$ que la mutation isolée est la seule responsable du phénotype observé. Seul le modèle des souris knock-out $(\mathrm{KO})$ permet cela.

Les souris $\mathrm{KO}$ sont des animaux génétiquement modifiés par l'homme chez qui un gène a été supprimé ou invalidé. La connaissance complète du génome de la souris publiée en 2003 permet actuellement de créer facilement sur demande une souris $\mathrm{KO}$ pour un gène de son choix. Dans le domaine de l'infectiologie et du sepsis, il existe un grand nombre d'exemples d'animaux $\mathrm{KO}$ susceptibles à un pathogène donné et permettant de faire le lien entre ce gène et un tableau clinique. Par exemple, Cooper et al. démontrent que des souris $\mathrm{KO}$ pour le gène d'une sous-unité de l'IL-12 ou du récepteur à l'interféron gamma présentent une susceptibilité aux infections à mycobactéries $(2,3)$. Cela souligne le rôle majeur de ces deux cytokines dans la défense contre les germes intracellulaires. On peut aussi citer les souris KO pour TLR4 qui sont résistantes au LPS, confirmant les expériences de Poltorak citées précédemment où les souris $\mathrm{KO}$ pour Toll2, un autre membre de la famille des récepteurs Toll, sont sensibles aux bactéries à Gram positif et aux infections à Candida.

\section{Les études humaines}

De très nombreuses études épidémiologiques réalisées au sein de populations d'adoptés, de jumeaux ou d'ethnies différentes ont confirmé chez l'homme cette inégalité génétique face au risque infectieux.

Sorensen $e t$ al. ont évalué l'influence des facteurs génétiques et environnementaux sur le risque de décès prématuré (avant 50 ans) chez 1000 enfants adoptés. Le risque relatif pour un enfant de mourir prématurément d'infection est de 5,8 si un de ses parents biologiques (avec qui il partage son patrimoine génétique) est décédé prématurément d'infection, alors que ce risque est de 0,73 si un de ses parents adoptifs (avec qui il partage son " environnement") décède des suites d'une infection. Cette étude démontrait aussi que les facteurs génétiques ont un rôle prédominant pour les maladies cardio-vasculaires (risque relatif 3,8 ) et pour toutes causes confondues, mais de façon moins nette (risque relatif 1,4$)(4)$. 
Les études de jumeaux permettent également de mieux comprendre l'importance des facteurs génétiques par rapport aux facteurs environnementaux. Ces études évaluent le risque pour un jumeau d'être malade quand l'autre jumeau est atteint. En comparant ce risque entre des paires de jumeaux homozygotes (qui ont le même matériel génétique) et hétérozygotes (qui partagent au maximum $50 \%$ de leur génome) on a le poids des gènes dans ce risque. Ainsi, si un jumeau est atteint d'une pathologie infectieuse, il est clairement démontré que le risque de développer cette même pathologie chez l'autre jumeau est plus élevé pour des paires homozygotes que pour des jumeaux hétérozygotes. Cela a été rapporté dans plusieurs études sur la tuberculose (5), la lèpre (6), Helicobacter pilori (7), Plasmodium falciparum (8) et HIV-1 (9).

Enfin les études d'ethnies ou de races différentes vivant dans le même milieu retrouvent des incidences différentes de certaines maladies infectieuses comme le paludisme et la tuberculose.

Toutes ces études prouvent que la réponse anti-infectieuse peut être modifiée par l'altération d'un seul gène et que la part génétique dans le risque infectieux est supérieure à l'environnement. Les maladies génétiques en cause sont classées en deux catégories : maladies mendéliennes et maladies polygéniques.

\section{Les maladies génétiques mendéliennes}

Elles sont responsables d'infections sévères dès la petite enfance et sont transmises selon un mode de transmission récessif ou dominant. Elle doivent être systématiquement recherchées en cas d'infections répétées à germes banals ou peu pathogènes, telles que les arthrites répétées à pneumocoques, les gingivites, etc. Elles ne seront pas traitées dans le cadre de ce chapitre, mais on peut citer rapidement deux excellentes revues récentes sur les grandes anomalies impliquées : déficits primaires des lymphocytes (10), déficits primaires des phagocytes (11). D'autres déficits plus rares existent : déficits du complément responsables d'infections à germes encapsulés, déficits des voies de signalisation intracellulaires (interféron gamma et IL-12 augmentant le risque aux Mycobactéries, mutations de la kinase IRAK).

\section{Sepsis sévère : maladie polygénique}

Comme l'hypertension artérielle, le diabète, la maladie coronaire et quasiment l'ensemble des pathologies médicales, le sepsis sévère peut être considéré comme une maladie polygénique, c'est-à-dire que des variabilités de plusieurs gènes permettent d'expliquer la survenue ou la sévérité d'un tableau infectieux chez un individu donné ( $c f$. tableau I). Ces variabilités sont appelées polymorphisme génétique quand la maladie est présente chez plus de $1 \%$ de la population. Il s'agit souvent du changement d'une seule base (SNPs : single 
nucleotide polymorphisms), de la répétition en nombre variable de la même séquence d'ADN (VNTRs : variable number tandem repeats ou microsatellite) ou de l'absence d'une partie du gène (insertion/délétion). L'analyse du génome humain a montré l'existence de plus de 4 millions de SNPs. La plupart des polymorphismes génétiques sont sans effet sur le phénotype, car situés dans des régions non codantes (introns, régions inter-géniques) ou dans des exons, mais ne changeant pas l'acide aminé (par exemple les séquences TCA et TCC donnent une sérine). Compte tenu de la taille du génome humain, les études se sont essentiellement intéressées à des polymorphismes au sein ou à proximité de gènes de protéines impliquées dans la physiopathologie du sepsis. Ces études ont posé deux grandes questions : existe-t-il des marqueurs génétiques associés à un risque de développer un sepsis ? Existe-t-il des marqueurs génétiques associés au pronostic du sepsis?

Tableau I - Polymorphismes des gènes associés avec une susceptibilité au sepsis ou avec son pronostic.

\begin{tabular}{|c|c|c|c|c|}
\hline Gène & Chromosome & Polymorphisme & Clinique/association & Réf. \\
\hline LBP & $20 \mathrm{q} 11.23-\mathrm{q} 12$ & Cys98Gly & Sepsis (hommes) & 17 \\
\hline CD14 & $5 \mathrm{q} 31.1$ & C-159T & Sepsis, mortalité (homozygotes TT) & $\begin{array}{l}21 \\
22\end{array}$ \\
\hline TLR4 & $9 \mathrm{q} 32-\mathrm{q} 33$ & Asp299Gly & Sepsis Gram -, méningocoque & $\begin{array}{l}18, \\
39\end{array}$ \\
\hline TLR2 & $4 \mathrm{q} 32$ & $\operatorname{Arg} 753 \mathrm{Gln}$ & Sepsis Gram + & 19 \\
\hline MBL & $10 \mathrm{q} 11.2-\mathrm{q} 21$ & $\begin{array}{l}\text { Variants B,C,D } \\
\text { (codons 52, 54, 57) }\end{array}$ & Sepsis, mortalité, méningocoque & $\begin{array}{l}13- \\
16, \\
39\end{array}$ \\
\hline $\begin{array}{l}\text { FcgRIIa } \\
\text { FcgRIIIa } \\
\text { FcgRIIIb }\end{array}$ & $\begin{array}{l}1 \mathrm{q} 21 \\
1 \mathrm{q} 23 \\
1 \mathrm{q} 23\end{array}$ & $\begin{array}{l}\text { His131Arg } \\
\text { Val158Phe } \\
\text { NA1/NA2 }\end{array}$ & $\begin{array}{l}\text { Méningocoque, pneumocoque, } \\
\text { mortalité }\end{array}$ & $\begin{array}{l}23 \\
24, \\
39\end{array}$ \\
\hline Caspase 12 & $211 \mathrm{q} 22.3$ & Csp12-L & Sepsis (Africains) & 25 \\
\hline TNF- $\alpha$ & $6 \mathrm{p} 21.3-\mathrm{p} 21.1$ & G-308A & $\begin{array}{l}\text { Méningocoque, neuro-paludisme, } \\
\text { leishmaniose, sepsis, mortalité } \\
\text { (TNF2) }\end{array}$ & $\begin{array}{l}31 \\
32 \\
39\end{array}$ \\
\hline TNF $\beta$ & $6 \mathrm{p} 21.3$ & Ncol & Sepsis, mortalité (TNFB2) & 33 \\
\hline IL-1-raA2 & $2 \mathrm{q} 14.2$ & 86bpVNTR & Sepsis & 28 \\
\hline IL-10 & $1 \mathrm{q} 31-32$ & C-592A & Sepsis, mortalité & 30 \\
\hline IL-6 & $7 \mathrm{p} 21$ & G-174C & Méningocoque & $\begin{array}{l}29 \\
39\end{array}$ \\
\hline $\begin{array}{l}\text { IFN- } \gamma \\
\text { IFN- } \gamma \mathrm{R}\end{array}$ & $\begin{array}{l}12 \mathrm{q} 14 \\
6 \mathrm{q} 23-\mathrm{q} 24\end{array}$ & $\begin{array}{l}\text { IFN- } \gamma \text { VNTR } \\
\text { IFN } \gamma R \text { VNTR }\end{array}$ & Infection, sepsis & 34 \\
\hline PAI-1 & $7 \mathrm{q} 22.1-\mathrm{q} 22.3$ & 4G/5G insertion/délétion & $\begin{array}{l}\text { Méningocoque, sepsis, mortalité } \\
(4 \mathrm{G} / 4 \mathrm{G})\end{array}$ & $\begin{array}{l}36- \\
39\end{array}$ \\
\hline $\begin{array}{l}\text { Fact. } \\
\text { V Leiden }\end{array}$ & $1 q 23$ & FVLG1691A & Méningocoque, sepsis & $\begin{array}{l}39, \\
40\end{array}$ \\
\hline ECA & $17 \mathrm{q} 23$ & 287bp insertion/délétion & Mortalité, méningocoque & 41 \\
\hline
\end{tabular}


Les polymorphismes pour lesquels une réponse à ces questions a été obtenue concernent actuellement des gènes de protéines impliquées dans la détection des pathogènes et dans la signalisation intracellulaire, les cytokines pro- et anti-inflammatoires et les médiateurs de la coagulation $(12,26,27)$.

\section{Variation de la détection des pathogènes}

Les maladies infectieuses sont uniques en médecine car elles font intervenir des micro-organismes vivants qui ont un temps de doublement court (de l'ordre de dix-huit minutes). Ainsi, tout défaut de reconnaissance immédiate de "l'agresseur " conduira à sa multiplication et, à terme, à un challenge infectieux plus sévère. De nombreuses molécules plasmatiques et membranaires ont un rôle clé dans la reconnaissance des pathogènes.

\section{La mannose-binding lectine (MBL)}

La MBL est une protéine plasmatique de la phase aiguë de l'inflammation sécrétée par le foie. Elle appartient à la famille des collectines. Elle se lie aux sucres présents à la surface des bactéries, des levures et de certains virus et parasites. Elle agit, soit en déclenchant la phagocytose en opsonisant le micro-organisme, soit par toxicité directe via le complexe d'attaque membranaire du complément. Le gène de la MBL a trois polymorphismes au niveau de l'exon 1 qui entraînent des substitutions d'acides aminés au niveau des codons 52,54 et 57 . Il existe également un polymorphisme au niveau du promoteur du gène de la $\mathrm{MBL}$; la guanine est remplacée par la cytosine en position -221 (G-221C). Les individus hétérozygotes pour ces polymorphismes ont des taux diminués de MBL, alors que les variants homozygotes et les hétérozygotes composites (associant plusieurs modifications) ont des taux quasi nuls de MBL plasmatique. Garred et al. ont étudié le polymorphisme de la MBL chez 272 patients de réanimation polyvalente ayant un SIRS. La présence d'un variant de la MBL est associée avec la survenue d'un sepsis ou d'un sepsis sévère et avec une surmortalité (13). Par ailleurs, plusieurs études chez des patients atteints de cancers ont souligné la susceptibilité aux infections sévères chez les patients aplasiques porteurs de ces variants $(14,15)$. Enfin, Roy et al. ont montré que les patients homozygotes pour l'un des polymorphismes de la MBL ont un risque relatif 3,5 fois plus élevé de développer une infection grave à pneumocoque, ce qui est équivalent au risque rapporté après splénectomie (16). Il serait donc envisageable de détecter ces polymorphismes dans la population générale pour proposer une vaccination anti-pneumococcique chez ces sujets à haut risque. 


\section{La lipopolysaccaride binding protein (LBP) et la bactericidal permeability increasing protein (BPI)}

Quand le LPS est libéré dans le sang par les bactéries à Gram négatif (soit spontanément soit après action des antibiotiques), il se lie, soit à la LBP, qui est une protéine plasmatique de la phase aiguë qui le présente ensuite au récepteur CD14 permettant l'activation des macrophages, soit au BPI, qui est une protéine produite par les polynucléaires, qui inhibe la liaison du LPS au CD14 et à TLR4. Trois polymorphismes de la BPI et deux polymorphismes de la LBP ont été étudiés chez 204 patients septiques. L'allèle Cys98Gly (remplacement de la cystéine par la glycine en position 98) est plus fréquent chez les hommes atteints de sepsis par rapport à la population contrôle (17).

\section{Les récepteurs de type Toll (Toll-Like Receptor, TLR)}

On connaît actuellement dix récepteurs de type Toll (TLR1 à TLR10). Ils reconnaissent spécifiquement différents composants des micro-organismes et leur stimulation conduit au développement de la réaction inflammatoire en activant, par exemple, le facteur transcriptionnel $N F-\kappa B$ qui est un élément central de la synthèse des principales cytokines pro-inflammatoires.

Comme rapporté précédemment, TLR4 reconnaît le LPS des bactéries à Gram négatif. L'étude du gène de TLR4 a retrouvé deux polymorphismes dans sa partie codante : la substitution d'une adénine en 896 par une guanine conduit au remplacement d'un acide aspartique par une glycine (Asp299Gly). Un autre polymorphisme conduit au remplacement de la thréonine 399 par une isoleucine (Thr399Ile) également dans la partie extracellulaire du récepteur, c'est-à-dire dans celle impliquée dans la reconnaissance du complexe LPS/LBP/CD14. Lorenz et al. ont décrit la fréquence de ces deux polymorphismes dans un groupe de 91 patients présentant un choc septique. Ils ne retrouvent pas de différence par rapport au groupe contrôle pour le double polymorphisme Asp299Gly/Tre399Ile. Par contre, 5 patients en choc septique présentent de façon isolée le polymorphisme Asp299Gly contre 0 dans le groupe contrôle. Enfin, si on considère uniquement les sepsis à Gram négatif, qui sont les seuls à pouvoir être influencés par ces polymorphismes, 29\% de ces patients sont porteurs de variants TLR 4 contre $10 \%$ de la population contrôle (18). À l'inverse, ce polymorphisme Asp299Gly n'a été associé ni à la susceptibilité ni à la sévérité d'infections à méningocoque (20).

TLR2 reconnaît les bactéries à Gram positif, les mycobactéries et les levures. Lorenz et al. ont mis en évidence un polymorphisme de TLR2 : Arg753Gln (substitution de l'arginine située en position 753 de la séquence protéique par une glutamine). In vitro, les cellules transfectées avec ce récepteur muté ont une moindre réponse lors de la stimulation par des peptides bactériens dérivés de Borrelia burgdorferi et de Treponema pallidum. Létude des 91 patients précédemment étudiés pour TLR4 retrouve deux patients hétérozygotes pour Arg753Gln 
présentant une infection à staphylocoque (19). Même si l'effectif est faible, il est clair que l'étude de ce polymorphisme peut être intéressant dans une population de choc septique à Gram positif. Récemment ce polymorphisme a aussi été associé à un risque élevé de tuberculose dans une population tunisienne.

\section{Le récepteur CD14}

Il est présent sous deux formes :

- une glycoprotéine membranaire (exprimée principalement à la surface des macrophages, des monocytes et des polynucléaires), qui se lie en particulier au LPS, au peptidoglycane et à l'acide lipotéichoïque ;

- une forme soluble, qui permet l'activation par le LPS des cellules CD14 négatives.

Il existe un polymorphisme fonctionnel du promoteur de CD14 : C-159T (la cytosine située en position -159 par rapport au début de la transcription, est remplacée par une thymine). Les porteurs de l'allèle $T$ ont des taux plus élevés de CD14 membranaires et solubles. Gibot et al. retrouvent une association entre les patients homozygotes TT et le risque de développer un choc septique (21). De plus, les patients TT ont un risque relatif de décès plus élevé (odds ratio : 5,3). Ce résultat n'a pas été retrouvé dans une étude allemande portant sur des sepsis sévères (22).

\section{Les récepteurs des immunoglobulines ( $F(\gamma R)$}

Ils sont présents à la surface des leucocytes et dans le plasma. Ils peuvent activer le complément, faciliter la phagocytose et médier la cytotoxicité anticorps-

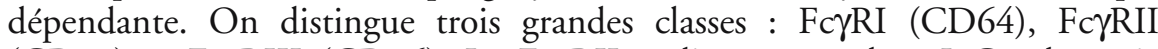
(CD32) et FcyRIII (CD16). Le FcyRII se lie au complexe IgG2a-bactérie encapsulée (Hamophilus influenza, Neisseria meningitidis, Streptococcus pneumonia) permettant la phagocytose et la destruction du pathogène. Il existe un polymorphisme du gène FcyRIIa qui modifie une histidine de la région extracellulaire impliquée dans la liaison de l'IgG2a et qui conduit à deux types de récepteurs : FcyRIIa-R131 et FcyRIIa-H131. Les patients homozygotes R/R131 (25\% de la population caucasienne) ne peuvent pas phagocyter les bactéries encapsulées via IgG2 et Fc $\gamma$ RIIa et sont plus susceptibles aux infections graves à méningocoque (23), ainsi qu'aux pneumopathies graves à pneumocoque (24).

\section{Polymorphismes modifiant la signalisation intracellulaire}

À ce jour, il existe un seul polymorphisme connu associant le sepsis et les molécules impliquées dans la signalisation intracellulaire. Les caspases sont des protéases intracellulaires impliquées dans l'apoptose ou dans l'inflammation 
(maturation des cytokines). La caspase 12 présente un polymorphisme au niveau de l'exon 4 de son gène, dans lequel la séquence TGA, donnant un codon stop en position 125, est remplacée par la séquence CGA codant pour une arginine. Cela donne naissance à deux protéines : une protéine tronquée Csp12-S (forme fréquente) et une pro-enzyme de taille normale, Csp12-L, retrouvée seulement chez les sujets d'origine africaine. La présence de l'allèle Csp12-L est associée à une diminution de la réponse inflammatoire après stimulation du sang total par le LPS ex vivo. Des études in vitro montrent que Csp12-L inhibe l'immunité innée et la réponse inflammatoire en agissant sur NF- $\kappa B$. Cet allèle est plus fréquent chez les Américains descendant d'Africains présentant un sepsis sévère (25).

\section{Polymorphismes affectant la réponse à l'infection}

\section{Les cytokines pro-inflammatoires et anti-inflammatoires}

\section{L'interleukine 1 (IL-1)}

L'IL-1 est une cytokine pro-inflammatoire sécrétée principalement par les macrophages. Elle est présente sous deux formes, l'IL-1 $\alpha$ et l'IL-1 $\beta$, qui se lient au même récepteur et ont les mêmes fonctions. Le gène de l'IL-1 $\beta$ est situé au sein d'un locus qui comprend aussi les gènes de l'IL-1 $\alpha$ et de l'IL-1 ra. L'IL-1 ra (IL-1 receptor antagonist) est une protéine de la phase aiguë qui se lie de façon compétitive au récepteur de l'IL-1. On décrit plusieurs polymorphismes au niveau de l'intron 2 de ce gène, donnant naissance à cinq allèles (IL-1 ra A1 à $\mathrm{A} 5$ ) et correspondant à deux, trois, quatre, cinq ou six copies d'une séquence de 86 paires de bases (86bp VNTR). L'allèle IL-1 ra A2 (deux copies) est présent chez $25 \%$ de la population et est associé à des taux supérieurs d'IL-1 ra à l'état de base et après stimulation, ce qui crée un état anti-inflammatoire. Fang et al. retrouvent une augmentation du risque de sepsis sévère chez les patients $\mathrm{A} 2$, avec un risque relatif à 2,14 pour les homozygotes et à 1,73 pour les hétérozygotes, sans lien avec la mortalité (28). On connaît par ailleurs deux polymorphismes de l'IL-1 $\beta$ (AvaI et TaqI) et un polymorphisme de l'IL-1 $\alpha$, dont les conséquences sur la fonction des protéines ne sont pas claires, et pour lesquels aucune association n'a été démontrée.

\section{L'interleukine 6 (IL-6)}

L'IL-6 est une cytokine pro-inflammatoire. Il existe un polymorphisme de son promoteur, G-174C (substitution de la guanine par la cytosine). Les patients homozygotes GG semblent avoir un meilleur pronostic de leur sepsis sévère, bien que ce génotype ne semble pas associé avec le taux d'IL-6 (29). 


\section{L'interleukine 10 (IL-10)}

L'Il-10 est une cytokine anti-inflammatoire sécrétée principalement par les monocytes et les macrophages. On décrit cinq polymorphismes dans la région du promoteur de l'IL-10 : trois SNP (G-1082A, C-819T et C-592A), et deux répétitions de nucléotides (CA) en -1151 et en -3978 . Lowe et al. ont étudié ces polymorphismes chez des patients de réanimation. L'allèle C-592A est associé à des taux plus bas d'IL-10 après stimulation, à une susceptibilité au sepsis et à une augmentation de la mortalité (30). Des résultats identiques ont été rapportés chez plus de 1000 enfants atteints de méningococcémies.

\section{Le tumor necrosis factor alpha (TNF- $\alpha$ )}

Le TNF- $\alpha$ est la cytokine pro-inflammatoire centrale, sécrétée principalement par les monocytes dès le début de la réaction inflammatoire. Son promoteur contient de nombreux polymorphismes $(-238,-308,-476)$ qui ont été associés à différentes pathologies infectieuses. Le polymorphisme G-308A a été particulièrement étudié. La guanine $\mathrm{G}$ correspondant à l'allèle TNF1 est remplacée par l'adénine A correspondant à l'allèle TNF2 (fréquence de $20 \%$ chez les Caucasiens). Les individus possédant un ou deux allèles TNF2 ont un taux de TNF plus élevé à l'état de base et après stimulation. Dans le choc septique, TNF2 a été associé à un risque accru de développer ce syndrome (39\% des patients possèdent l'allèle TNF2 contre $18 \%$ dans le groupe contrôle) et à une surmortalité. L'allèle TNF2 est présent chez $52 \%$ des décédés contre $24 \%$ des survivants. A SAPS II égal, les patients TNF2 (homozygotes ou hétérozygotes) ont 3,7 fois plus de risque de décéder (31). Tang et al. retrouvent une augmentation de la mortalité chez des Taïwanais TNF2 présentant un choc septique en postopératoire (32). D'autres études montrent que les patients porteurs de l'allèle TNF2 ont un risque multiplié par 7 de faire un neuro-paludisme mortel, par 3,5 de faire une forme sévère de leishmaniose, et par 2,5 fois de décéder au cours d'une méningococcie.

Le gène du TNF- $\beta$ est situé en amont, à proximité de celui du TNF $\alpha$. Il existe un polymorphisme du premier intron du TNF- $\beta$ en position 1069 qui a été associé à une forte production de TNF $\alpha$. L'allèle commun TNFB2 (fréquence de $70 \%$ ) contient une adénine, alors que l'allèle TNFB1 possède une guanine. Dans une population de sepsis sévère, TNFB2 (homozygote) est retrouvé chez $80 \%$ des décédés contre $40 \%$ des survivants (33). L'étude de 110 patients présentant un traumatisme sévère montre par ailleurs que les homozygotes TNFB2 présentent un risque supérieur de développer un sepsis (odds ratio : 5,22).

\section{L'interféron gamma (IFN- $\gamma$ )}

L'IFN- $\gamma$ est produit par les lymphocytes NK et les lymphocytes T. Il stimule l'expression des molécules de classe II du CMH (complexe majeur d'histocompatibilité) et la production de TNF- $\alpha$ et d'IL-1. On connaît des 
polymorphismes du gène de l'IFN- $\gamma$ et de son récepteur IFN- $\gamma$ R1 (IFN- $\gamma$ receptor 1). Davis et al. décrivent une association de certains allèles de ce récepteur avec la survenue d'infections sévères chez des patients après un polytraumatisme (34).

\section{L'activation et l'inhibition de la coagulation}

La coagulation est une part importante de la réponse de l'organisme aux pathogènes, et pourrait permettre de circonscrire les lieux de l'infection et limiter ainsi son extension. Cependant, une activation exagérée de la coagulation peut avoir des effets délétères en entraînant des défaillances d'organes. Il existe de très nombreux polymorphismes fonctionnels de la coagulation, mais, curieusement, seuls deux d'entre eux ont été étudiés dans le sepsis (35).

\section{Plasminogen activator inhibitor 1 (PAl-1)}

L'inhibiteur 1 de l'activateur du plasminogène est une protéine qui est sécrétée principalement par les cellules endothéliales, les hépatocytes et les plaquettes, sous la stimulation de cytokines pro-inflammatoires. Elle entraîne un état procoagulant par inhibition de la fibrinolyse naturelle. Il existe un polymorphisme $\mathrm{du}$ promoteur de son gène correspondant à l'insertion ou la délétion d'une guanine $(\mathrm{G})$ au sein d'une séquence GGGGG créant deux allèles appelés 4G et $5 \mathrm{G}$ en fonction de la délétion d'une base $\mathrm{G}$ ou de sa présence. Dans une étude portant sur 175 enfants présentant un sepsis à méningocoque, les sujets 4G/4G (homozygotes) ont un taux plus élevé de PAI-1 et une surmortalité (36). Cependant, elle ne met pas en évidence d'association entre le polymorphisme génétique et la susceptibilité pour cette infection grave. Westendorp et al. confirment ces résultats et montrent que les enfants de parents homozygotes $4 \mathrm{G}$ ont un risque six fois plus élevé de développer un sepsis à méningocoque qu'une méningite (37). Des résultats identiques ont été obtenus sur une cohorte de patients avec un polytraumatisme et parmi lesquels les sujets $4 \mathrm{G} / 4 \mathrm{G}$ ont des taux plus élevés d'IL-1, de TNF- $\alpha$ et de PAI-1 plasmatique, et un risque accru de sepsis, de défaillance d'organes et de mortalité (38).

\section{Le facteur $V$ Leiden}

Le facteur Va associé au facteur Xa se lient dans le complexe prothrombinase pour activer la prothrombine en thrombine (facteur IIa). La protéine $\mathrm{C}$ activée a un rôle d'anticoagulant en dégradant le facteur $\mathrm{V}$ au niveau de trois sites ( $\arg 306, \arg 506$ et $\arg 679)$. On décrit trois polymorphismes du gène du facteur V : le facteur V Cambridge (Arg306Thr), le facteur V HongKong (Arg306Gly) et le facteur V Leiden (Arg506Gln présent chez 5 à $10 \%$ des Caucasiens). Ces mutations ont un effet pro-coagulant et représentent un facteur de risque reconnu de thrombose veineuse. Létude de 259 enfants atteints de méningococcie n'a pas révélé d'association entre le facteur $V$ Leiden et la susceptibilité ou la mortalité. Cependant, les patients hétérozygotes 
présentent un nombre accru de complications à type de nécroses cutanées et amputations (39). À l'inverse, Kerlin et al. rapportent que les patients hétérozygotes pour le facteur $\mathrm{V}$ Leiden ont une meilleure survie dans le sepsis sévère et bénéficient tout autant de l'administration de protéine $\mathrm{C}$ recombinante que les patients non porteurs de la mutation (40). Ce dernier point suggère que les mécanismes d'action de la PCA dans le sepsis ne sont pas uniquement liés à ses effets anticoagulants.

\section{Enzyme de conversion de l'angiotensine (ECA)}

C'est une glycoprotéine synthétisée principalement par les cellules endothéliales et certaines cellules épithéliales. Elle possède une forme membranaire et une forme plasmatique. Elle transforme l'angiotensine I en angiotensine II et dégrade de nombreux métabolites dont la bradykinine, les peptides neuronaux, les facteurs chimiotactiques. De façon intéressante, cette enzyme a un rôle central et limitant dans la synthèse de l'angiotensine, qui est elle-même un activateur puissant de la synthèse des radicaux libres. Il existe une insertion/délétion de 287 paires de bases au niveau d'une région non codante du gène de l'ACE donnant deux génotypes : D Délétion, I Insertion. Des études in vitro ont montré que l'allèle $\mathrm{D}$ est responsable d'une synthèse accrue de ACE, d'une production augmentée d'angiotensine II et de radicaux libres. Par ailleurs, le génotype DD a été associé avec la sévérité et la mortalité dans une population d'enfants présentant un sepsis à méningocoque, ainsi qu'avec un risque accru de développer un ARDS et d'en mourir dans un groupe de patients atteints de sepsis sévère (41).

\section{Perspectives}

La liste des polymorphismes génétiques associés à une susceptibilité à l'infection ou à une sévérité accrue des états septiques s'allonge régulièrement. Il reste encore de nombreux gènes candidats, soit dans les catégories déjà explorées, soit dans d'autres classes de protéines impliquées, par exemple dans le métabolisme, le cycle cellulaire, le transport protéique et, bien sûr, la pharmacogénomique. Outre la recherche de nouveaux polymorphismes, il reste aussi à évaluer le rôle de l'association de plusieurs polymorphismes chez le même patient, car le sepsis est influencé par les produits de nombreux gènes. Pour répondre à ces deux objectifs, il est indispensable d'analyser de larges populations pour valider les résultats et passer à une étape ultérieure qui sera préventive et thérapeutique (42).

Les polymorphismes génétiques ne sont pas toujours délétères et leur maintien au cours de l'évolution est certainement dû à un avantage sélectif. Par exemple, dans l'infection par le VIH, on rencontre des polymorphismes protecteurs qui, certes, n'empêchent pas la contamination, mais limitent la sévérité de la maladie (43). De même, on a vu que la mutation Leyden/pro- 
thrombogène est protectrice dans le sepsis, et il a été démontré que les variants de TLR4 diminuent le risque d'athérosclérose.

L'avenir est au traitement individuel, adapté au polymorphisme génétique de chaque patient, aussi bien dans la physiopathologie de la maladie que dans la pharmaco-génomique. Cela dépasse largement le cadre du sepsis pour englober les pathologies infectieuses en général, les maladies cardio-vasculaires, le cancer, la greffe d'organe, etc. Bien entendu, cela ne peut se faire sans une éducation renforcée des médecins dans ce domaine et cela nécessite une législation stricte afin que ces avancées médicales ne conduisent pas à une discrimination sociale ou professionnelle qui serait absurde, car il est important de rappeler qu'il ne s'agit que de facteurs de risque.

\section{Références}

1. Poltorak A, He X, Smirnova I et al. (1998) Defective LPS signaling in C3H/HeJ and C57BL/10ScCr mice: mutations in Tlr4 gene. Science 282: 2085-8

2. Cooper AM, Dalton DK, Stewart TA et al. (1993) Disseminated tuberculosis in interferon gamma gene-disrupted mice. J Exp Med 178: 2243-7

3. Cooper AM, Magram J, Ferrante J et al. (1997) Interleukin 12 (IL-12) is crucial to the development of protective immunity in mice intravenously infected with mycobacterium tuberculosis. J Exp Med 186: 39-45

4. Sorensen TI, Nielsen GG, Andersen PK et al. (1988) Genetic and environmental influences on premature death in adult adoptees. N Engl J Med 318: 727-32

5. Comstock GW (1978) Tuberculosis in twins: a re-analysis of the Prophit survey. Am Rev Respir Dis 117: 621-4

6. Fine PE (1981) Immunogenetics of susceptibility to leprosy, tuberculosis, and leishmaniasis. An epidemiological perspective. Int J Lepr Other Mycobact Dis 49: 437-54

7. Malaty HM, Graham DY, Isaksson I et al. (2000) Are genetic influences on peptic ulcer dependent or independent of genetic influences for influences for Helicobacter pylori infection? Arch Intern Med 160: 105-9

8. Jepson AP, Banya WA, Sisay-Joof F et al. (1995) Genetic regulation of fever in Plasmodium falciparum malaria in Gambian twin children. J Infect Dis 172(1) : 316-9

9. Chang J, Naif HM, Li S et al. (1996) Twin studies demonstrate a host cell genetic effect on productive human immunodeficiency virus infection of human monocytes and macrophages in vitro. Journal of Virology 70: 7792-803

10. Buckley RH (2000) Primary immunodeficiency diseases due to defects in lymphocytes. N Engl J Med 343: 1313-24

11. Andrews T, Sullivan KE (2003) Infections of patients with inherited defects in phagocytic function. Clin Microbiol Rev 16(4): 597-621

12. Lin MT, Albertson TE (2004) Genomic polymorphisms in sepsis. Crit Care Med 32(2): 569-79

13. Garred P, J Strom J, Quist L et al. (2003) Association of mannose-binding lectin polymorphisms with sepsis and fatal outcome, in patients with systemic inflammatory response syndrome. J Infect Dis 188(9): 1394-403

14. Neth O, Hann I, Turner MW et al. (2001) Deficiency of mannose-binding lectin and burden of infection in children with malignancy: a prospective study. Lancet 358: 614-8

15. Hibbert ML, Sumiya M, Summerfield JA et al. (1999) Association of variants of the gene for mannose-binding lectin with susceptibility to meningococcal disease. Meningococcal Research Group. Lancet 353: 1049-53 
16. Roy S, Knox K, Segal S et al. (2002) MBL genotype and risk of invasive pneumococcal disease: a case control study. Lancet 359: 1569-73

17. Hubacek JA, Stuber F, Frohlich D et al. (2001) Gene variants of the bactericidal/permeability increasing protein and lipopolysaccharide binding protein in sepsis patients: gender-specific genetic predisposition to sepsis. Crit Care Med 29: 557-61

18. Lorenz E, Mira JP, Frees KL et al. (2002) Relevance of mutations in the TLR4 receptor in patients with gram-negative septic shock. Arch Intern Med 162: 1028-32

19. Lorenz E, Mira JP,cornish KL et al. (2000) A novel polymorphism in the toll-like receptor 2 gene and its potential association with staphylococcal infection. Infect Immun 68: 6398-401

20. Read RC, Pullin J, GregoryS et al. (2001) A functional polymorphism of toll-like receptor 4 is not associated with likelihood or severity of meningococcal disease. J Infect Dis 184: 640-2

21. Gibot S, Cariou A, Drouet L et al. (2002) Association between a genomic polymorphism within the CD14 locus and septic shock susceptibility and mortality rate. Crit Care Med 30: 969-73

22. Hubacek JA, Stuber F, Frohlich D et al. (2000) The common functional C(-159)T polymorphism within the promoter region of the lipopolysaccharide receptor CD14 is not associated with sepsis development or mortality. Genes Immun 1: 405-7

23. Platonov AE, Shipulin GA, Vershinia IV et al. (1998) Association of human Fc gamma RIIa (CD32) polymorphism with susceptibility to and severity of meningococcal disease. Clin Infect Dis 27: 746-50

24. Yee AM, Phan HM, Zuniga R et al. (2000) Association between FcgammaRIIa-R131 allotype and bacteremic pneumococcal pneumonia. Clin Infect Dis 30: 25-8

25. Saleh M, Vaillancourt JP, Graham RK et al. (2004) Differential modulation of endotoxin responsivness by human caspase-12 polymorphisms. Nature 429(6987): 75-9

26. Tabrizi AR, Zehnbauer BA, Freeman BD et al. (2001) Genetic markers in sepsis. J Am Coll Surg 192: 106-17

27. Holmes CL, Russel JA, Walley KR (2003) Genetic polymorphisms in sepsis and septic shock: role in prognosis and potential for therapy. Chest 124(3): 1103-15

28. Fang XM, Schroder S, Hoeft A et al. (1999) Comparison of two polymorphisms of the interleukin-1 gene family: interleukin-1 receptor antagonist polymorphism contributes to susceptibility to severe sepsis. Crit Care Med 27: 1330-4

29. Schluter B, Raufhake C, Erren M et al. (2002) Effect of the interleukin-6 promoter polymorphism $(-174 \mathrm{G} / \mathrm{C})$ on the incidence and outcome of sepsis. Crit Care Med 30: 32-7

30. Lowe PR, Galley HF, Abdel-Fattah A et al. (2003) Influence of interleukin-10 polymorphisms on interleukin-10 expression and survival in critically ill patients . Crit Care Med 31: 34-8

31. Mira JP, Cariou A, Grall F et al. (1999) Association of TNF2, a TNF-alpha promoter polymorphism, with septic shock susceptibility and mortality : a multicenter study. JAMA 282: 561-8

32. Tang GJ, Huang SL, Yien HW et al. (2000) Tumor necrosis factor gene polymorphism and septic shock in surgical infection. Crit Care Med 28: 2733-6

33. Stüber F, Petersen M, Bokelmann F et al. (1996) A genomic polymorphism within the tumor necrosis factor locus influences plasma tumor necrosis factor-alpha concentrations and outcome of patients with severe sepsis. Crit Care Med 24: 381-4

34. Davis EG, Eichenberger MR, Grant BS et al. (2000) Microsatellite marker of interferongamma receptor 1 gene correlates with infection following major trauma. Surgery 128: $301-5$

35. Texereau J, Pene f, Chiche JD et al. (2004) Importance of hemostatic gene polymorphisms for susceptibility and outcome of severe sepsis. Crit Care Med 32 (5 suppl): S313-9

36. Hermans PW, Hibberd ML, Booy R et al. (1999) 4G/5G promoter polymorphism in the plasminogen-activator_inhibitor-1 gene and outcome of meningococcal disease. Meningococcal Research Group. Lancet 354: 556-60 
37. Westendorp RG, Hottenga JJ, Slagboom PE (1999) Variation in plasminogen-activatorinhibitor-1 gene and risk of meningococcal septic shock. Lancet 354: 561-3

38. Menges T, Hermans PW, Little SG et al. (2001) Plasminogen-activator-inhibitor-1 4G/5G promoter polymorphism and prognosis of severely injured patients. Lancet 357: 1096-7

39. Emonts M, Hazelzet JA, De Groot R et al. (2003) Host genetic determinants of Neisseria meningitidis infections. Lancet Infect Dis 3(9): 565-77

40. Kerlin BA, Yan SB, Isermann BH et al. (2003) Survival advantage associated with heterozygous factor $\mathrm{V}$ leiden mutation in patients with severe sepsis and in mouse endotoxemia. Blood 102: 3085-92

41. Harding D, Baines PB, Brull D et al. (2002) Severity of meningococcal disease in children and the angiotensin-converting enzyme gene polymorphism. Am J Respir Crit Care Med 165: 1103-6

42. Cariou A, Chiche JD, Charpentier J et al. (2002) The era of genomics: impact on sepsis clinical trial design. Crit Care Med 30: S341-8 